\title{
The Relationship Between Physical Development and Economic Growth in Japan: From the Perspective of Regional Differences in Biological Parameters
}

\author{
Yuki Kani $^{1,}$, , Katsunori Fujii ${ }^{2}$, Keiko Abe ${ }^{1}$, Kan-ichi Mimura ${ }^{1}$ \\ ${ }^{1}$ Faculty of Education, Osaka Seikei University, Osaka-city, Japan \\ ${ }^{2}$ Graduate School of Business Administration and Computer Science, Aichi Institute of Technology, Toyota-city, Japan \\ Email address: \\ kani@osaka-seikei.ac.jp (Y. Kani), fujii@aitech.ac.jp (Kastunori F.), abe-ke@osaka-seikei.ac.jp (Keiko A.), \\ mimura@osaka-seikei.ac.jp (Kan-ichi M.) \\ ${ }^{*}$ Corresponding author
}

\section{To cite this article:}

Yuki Kani, Katsunori Fujii, Keiko Abe, Kan-ichi Mimura. The Relationship Between Physical Development and Economic Growth in Japan: From the Perspective of Regional Differences in Biological Parameters. American Journal of Sports Science. Vol. 8, No. 4, 2020 , pp. 111-115. doi: $10.11648 /$ j.ajss.20200804.16

Received: December 2, 2020; Accepted: December 10, 2020; Published: December 22, 2020

\begin{abstract}
Following the Second World War, there was a rapid increase in body size in Japan as a result of qualitative changes in the human environment from marked economic growth and the stable supply of nutrition. A phenomenon that facilitated this physical growth may be seen in the larger body sizes and earlier maturation combined with the secular trends in GDP resulting from high economic growth. However, this high economic growth did not occur uniformly nationwide. Similarly in physical growth, the trend for earlier maturation was not seen uniformly nationwide. This study applied the wavelet interpolation method to identify the age at maximum peak velocity (MPV) in pubertal growth (a biological parameter) from the growth velocity curves for height described for the 47 prefectures of Japan from 1955 to 2015. A secular trend model of this biological parameter was then constructed. This parameter as seen particularly in urban and rural areas was then applied to the secular trend model and, based on a composition that eliminates regional differences, the possibilities as a barometer of economic growth based on the biological parameter of early maturation were then explored. The results showed a trend for earlier maturation in all prefectures for both boys and girls. The multi-year span evaluation chart in this study showed a trend for earlier maturation from 1955 to 1970 in Tokyo and other large cities, after which there was a transition to average maturation. In Aomori, Oita and other rural areas, there was a trend for late maturation from 1955 to 1970, followed by a transition to earlier maturation. From these trends, there may be said to be a close relationship between physical growth and socioeconomic status. However, since the late 1990s the difference in maturation rate in urban and rural regions has disappeared. This may be from the effect of high economic growth coming to an end.
\end{abstract}

Keywords: High Economic Growth, Wavelet Interpolation Model, Evaluation Chart

\section{Introduction}

The years from 1955 to 1973 are generally pointed to as Japan's period of high economic. Today, it is obvious that stable supply in the food situation and monumental improvements in the quality of nutrition have been behind the increase in physical size of Japanese people. After the Second World War, people's body size also increased rapidly in Japan, a phenomenon that was studied by Matsuura and Kawabata as an acceleration of physical growth or a promotion of growth
$[1,2]$. Kani et al. applied a cross-correlation function to the secular change curves of first largest peak velocity (FLPV) of physical growth in young children and GDP, and examined the similarities. Height in boys and girls was $\geq r=0.9$, showing a very high similarity. With these results it may be inferred that comparable trends would be seen in children of other ages as well [3]. The phenomenon of promoted physical growth, as if it were purely a result of high economic growth, may be said to show increasing body sizes and earlier maturation coupled with long-term trends in GDP. In fact, a major factor behind 
research on the promotion of growth in Japan is the school health statistical surveys that have been conducted in Japan from 1900 to the present. In this trend for improved physiques, physical development caught up with the prewar level in the late 1950s, but then continued to improve even after this catch-up phenomenon ended. This is called the growth acceleration phenomenon. It is characterized by accelerated growth during puberty due to larger body sizes, from which the age at which maximum rate of growth appears has also been assumed to be earlier. However, it was not clear whether younger age at maximum rate of growth had been verified. Kikuta et al. sought the age at which the annual amount of height growth was the highest, in other words, the age at peak height velocity (PHV) in puberty, and from analysis of the time trends in that age reported that the age at which PHV appeared was getting younger [4]. Fujii and Fujii et al. took this research a step further and identified the age at maximum peak velocity (MPV) in height using a wavelet interpolation model and verified the growth acceleration phenomenon from secular trends in age at MPV [5, 6]. However, in 1973, when Japan's high economic growth was coming to an end, the phenomenon of promoted growth was also gradually tapering off, and from that time until today no clear changes has been seen in the age at MPV of height. While it is certainly true that the average growth of physiques in Japan overall has not shown any marked changes and there is little fluctuation in age at MPV of height, a major pitfall exists in using averaged data for all of Japan. That is the lack of data collected from the same region every year. Consequently, there is a possibility that, depending on the case, major changes may be occurring in individual prefectures. Japan's period of high economic growth may have come to an end in 1973, but were those effects felt uniformly in all prefectures? It may be that there were large differences in the effects of high economic growth felt by each prefecture. However, we know nothing about those differences.

As post-war Japan entered a time of remarkable economic growth, environmental factors associated with food and nutrition also changed dramatically. As a result of those changes, the average height of boys in the final year of high school (17 years old) rose from $163.4 \mathrm{~cm}$ to $170.7 \mathrm{~cm}$, and that of girls from $153.2 \mathrm{~cm}$ to $158 \mathrm{~cm}$, from the 1955 to 2010 academic years. Those changes that occurred with high economic growth are thought to have had a huge effect on the bodies of Japanese. However, Morichi reported that this high economic growth was not necessarily uniform across the nation but rather was centered on major cities along the Pacific belt, and from there spread gradually to the regions [7]. Thus, it is unlikely that the trend for enhanced physical development occurred uniformly in cities and rural areas nationwide.

In this study, applying the wavelet interpolation model by Fujii [8], we identified the age at MPV in growth during puberty from height growth velocity curves described in each prefecture, investigated earlier age at MPV of height in prefectural units, and examined the relational composition of MPV with the socioeconomic status in each prefecture following the period of high economic growth.

\section{Methods}

\subsection{Data Sets}

The physical growth data used were height data for boys and girls from each prefecture for the years 1955 to 2015, obtained from school health statistical surveys published by the Ministry of Education, Culture, Sports, Science and Technology [9].

\subsection{Wavelet Interpolation Method (WIM)}

The theoretical background on the Wavelet Interpolation Model (WIM) and its effectiveness are described in a previous study by Fujii [10-13]. The Wavelet Interpolation Model (WIM) is a method to examine growth distance values at adolescent peak and menarchal age. A growth curve is produced by date-date interpolation with a wavelet function and by deriving the growth velocity curve obtained by differentiating the described distance curve to approximately describe the true growth curve from given growth date.

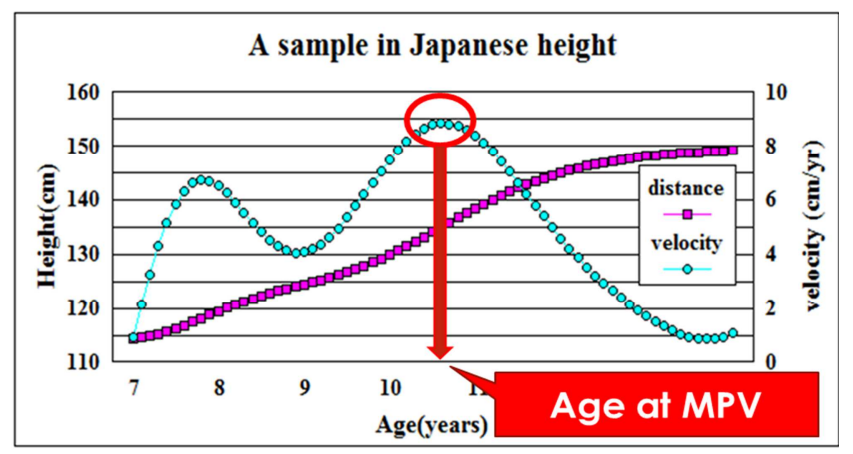

Figure 1. Wavelet Interpolation Method (WIM).

\subsection{Analytical Procedures}

1) Cohort data were assembled using height data for boys and girls for each prefecture from 1955 to 2015 published by the Ministry of Education, Culture, Sports, Science and Technology

2) Wavelet interpolation model was applied to the obtained cohort data to derive the annual trends in age at MPV in each prefecture.

3) Mean MPV age and standard deviation were calculated from height data for boys and girls from each prefecture.

4) Wavelet interpolation model was applied to the standard deviations of $\pm 0.5 \mathrm{SD}$ and $\pm 1.5 \mathrm{SD}$ from the mean age at MPV, and a 5-step evaluation chart was constructed.

5) The mean MPV ages for every five years in each prefecture were fit to the constructed 5-step evaluation chart. The relationship between that height growth and socioeconomic status in each prefecture after the period of high economic growth was investigated.

\subsection{Cohort}

In the present study, a cohort was referred to as a dataset for averaged growth for the same birth year. Because the same birth year was used as the base, the dataset was configured so that the secular trend and aging progress concurrently. 


\section{Results}

Investigation of the long-term trends in mean MPV age in each prefecture with application of the wavelet interpolation method showed a trend for earlier maturation in both boys (Figure 2) and girls (Figure 3).

Judging from multi-year span evaluation charts for each prefecture, prefectures that showed a trend for earlier maturation starting from an earlier time in boys were Hokkaido and Akita Prefectures in the Hokkaido/Tohoku region. Tokyo, Saitama, Chiba, and Kanagawa Prefectures in the Kanto region. Ishikawa, Fukui, Aichi and Mie Prefectures in the Hokuriku/Tokai region. Osaka and Hyogo Prefectures in the Kinki region. Fukuoka and Miyazaki Prefectures in the Kyushu/Okinawa region. In the Chugoku and Shikoku region, no prefectures showed trends for earlier maturation.

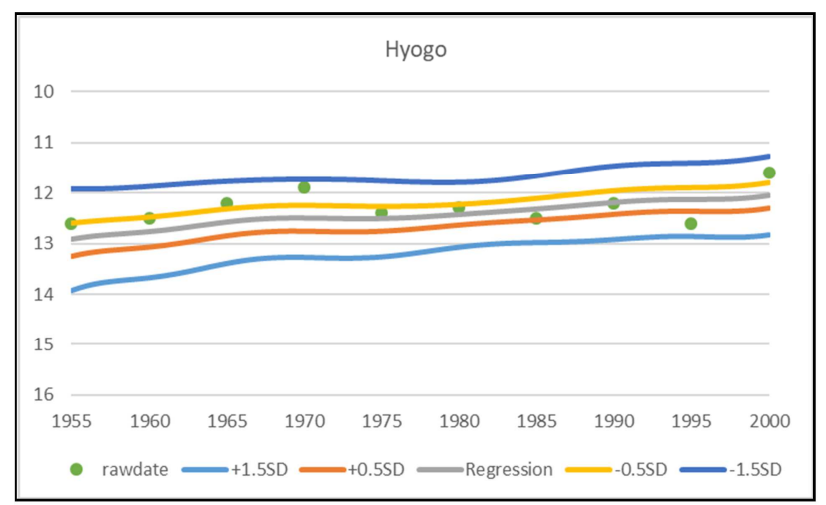

Figure 2. 5-step evaluation chart in boys.

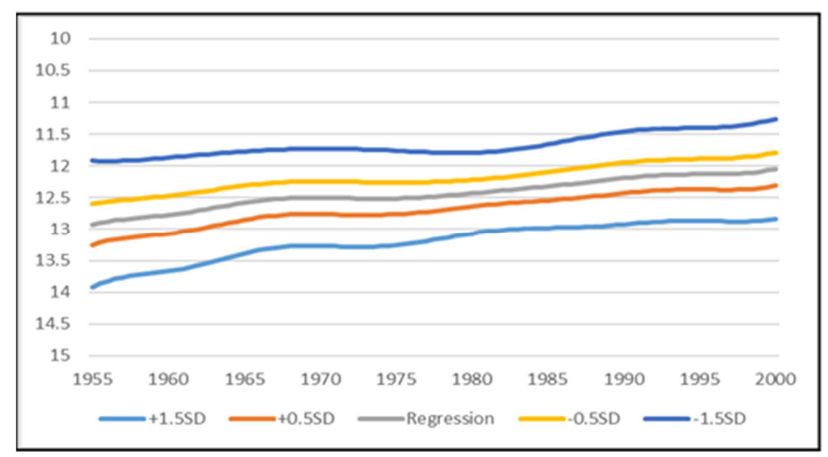

Figure 3. 5-step evaluation chart in girls.

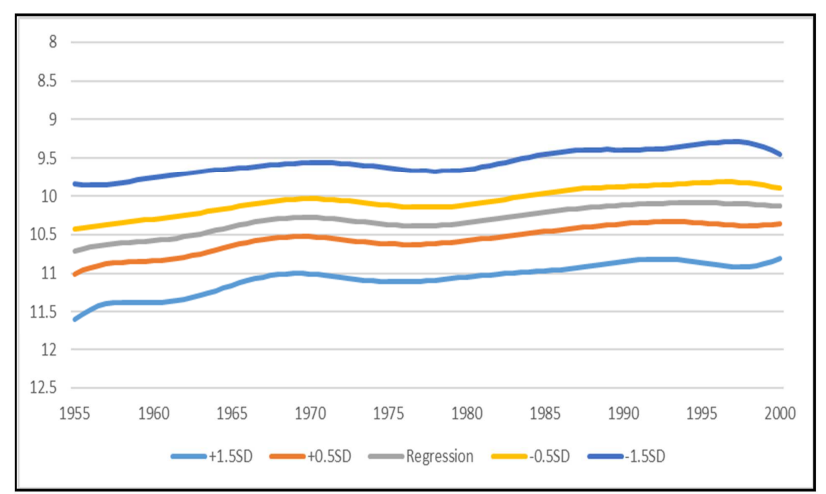

Figure 4. Investigation of the long-term trends in mean MPV age in boys (Tokyo).
Judging from multi-year span evaluation charts for each prefecture, in the Hokkaido/Tohoku region, no prefectures showed trends for earlier maturation in girls. Ibaraki, Gunma, Tokyo, Chiba, and Kanagawa Prefectures in the Kanto region. Ishikawa, Nagano, Yamanashi, Aichi and Mie Prefectures in the Hokuriku/Tokai region. Shiga, Osaka, Kyoto, Nara, Wakayama and Hyogo Prefectures in the Kinki region. Ehime, and Kochi Prefectures in the Shikoku region. Fukuoka, Saga and Nagasaki Prefectures in the Kyushu/Okinawa region. In the Chugoku region, no prefectures showed trends for earlier maturation.

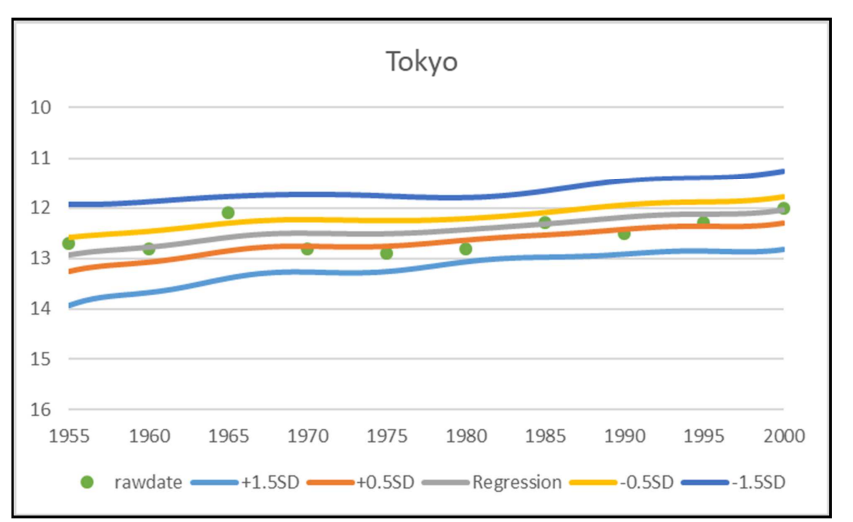

Figure 5. Investigation of the long-term trends in mean MPV age in boys (Hyogo).

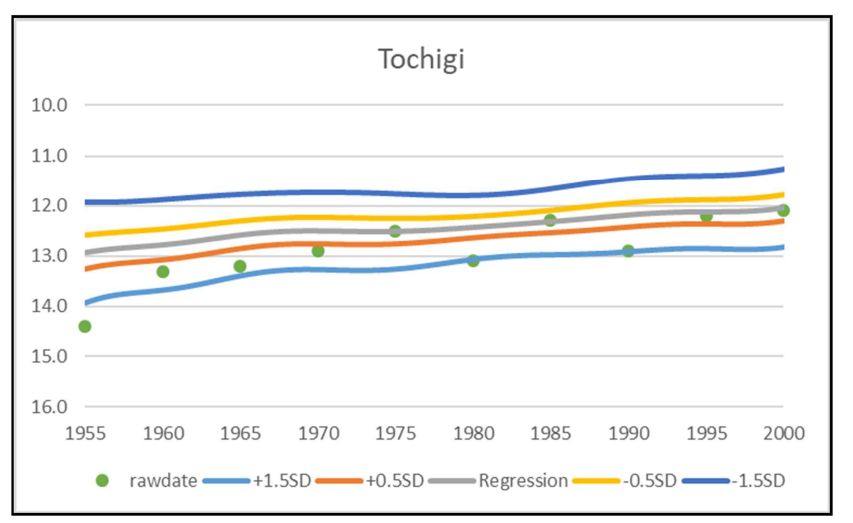

Figure 6. Investigation of the long-term trends in mean MPV age in boys (Tochigi).

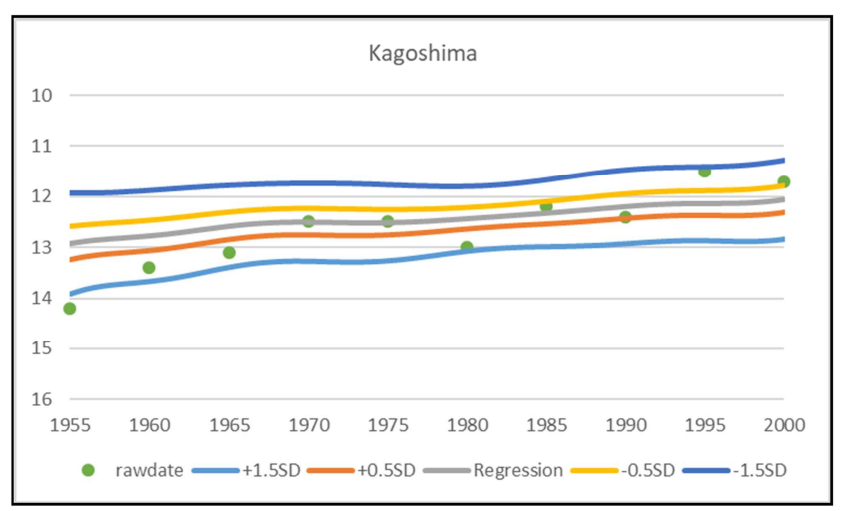

Figure 7. Investigation of the long-term trends in mean MPV age in boys (Kagoshima). 
Judging from multi-year span evaluation charts for each prefecture, in the Hokkaido/Tohoku region, no prefectures showed trends for earlier maturation in girls. Ibaraki, Gunma, Tokyo, Chiba, and Kanagawa Prefectures in the Kanto region. Ishikawa, Nagano, Yamanashi, Aichi and Mie Prefectures in the Hokuriku/Tokai region. Shiga, Osaka, Kyoto, Nara, Wakayama and Hyogo Prefectures in the Kinki region. Ehime, and Kochi Prefectures in the Shikoku region. Fukuoka, Saga and Prefectures in the Kyushu/Okinawa region. In the Chugoku region, no prefectures showed trends for earlier maturation.

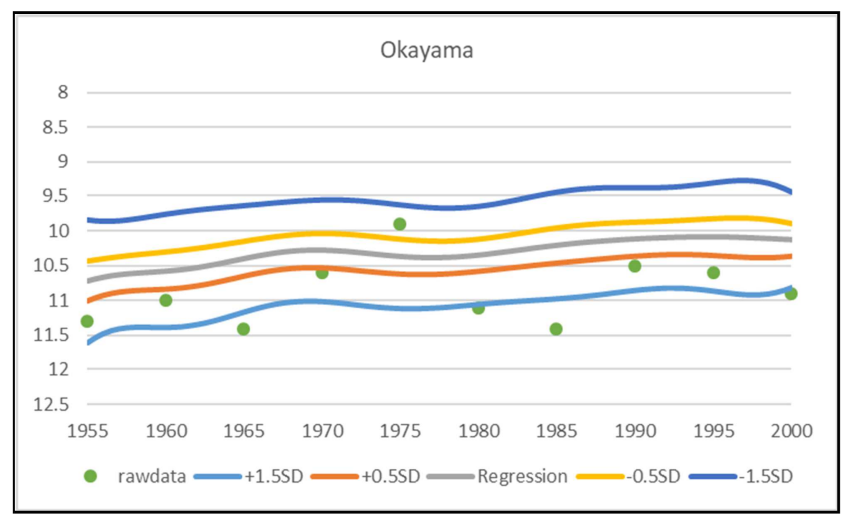

Figure 8. Investigation of the long-term trends in mean MPV age in girls (Tokyo).

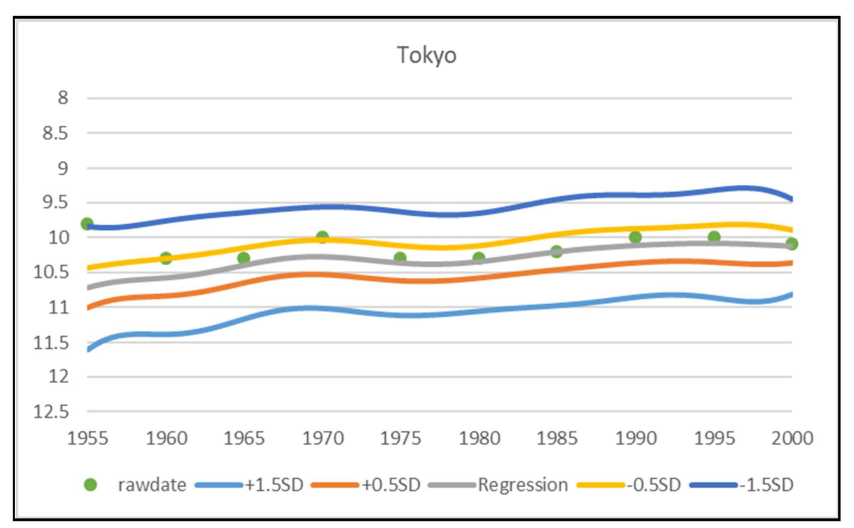

Figure 9. Investigation of the long-term trends in mean MPV age in girls (Fukuoka).

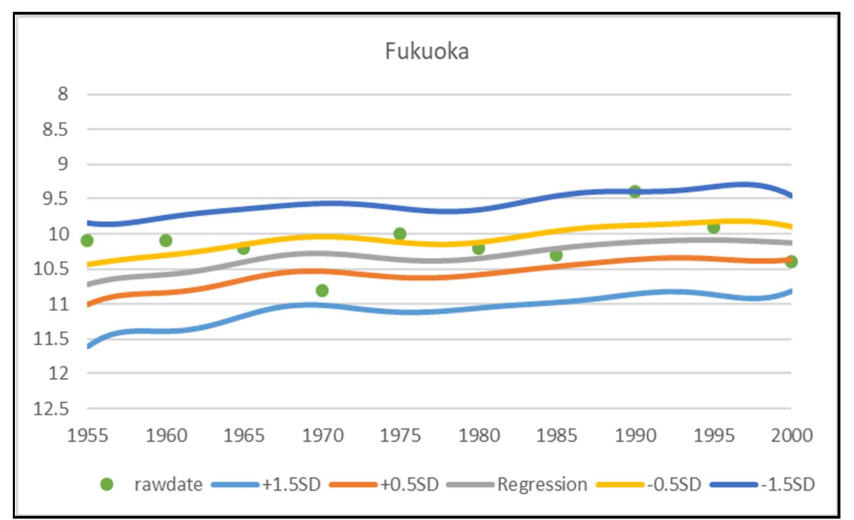

Figure 10. Investigation of the long-term trends in mean MPV age in girls (Okayama).
Prefectures that showed earlier maturation from a relatively late time similar to boys were Hokkaido and Aomori, Iwate, Akita, Yamagata, Miyagi, and Fukushima Prefectures in the Hokkaido/Tohoku region; Saitama Prefecture in the Kanto region; Niigata, Toyama, and Fukui Prefectures in the Hokuriku region; Gifu and Shizuoka Prefectures in the Chubu region; Okayama, Tottori, Hiroshima, Yamaguchi, Hiroshima, and Yamaguchi Prefectures in the Chugoku region; Tokushima and Kagawa Prefectures in the Shikoku region; and Oita, Nagasaki, Miyazaki, Kumamoto, Kagoshima, and Okinawa Prefectures in the Kyushu/Okinawa region. No prefectures in the Kinki region showed earlier maturation starting from a later time.

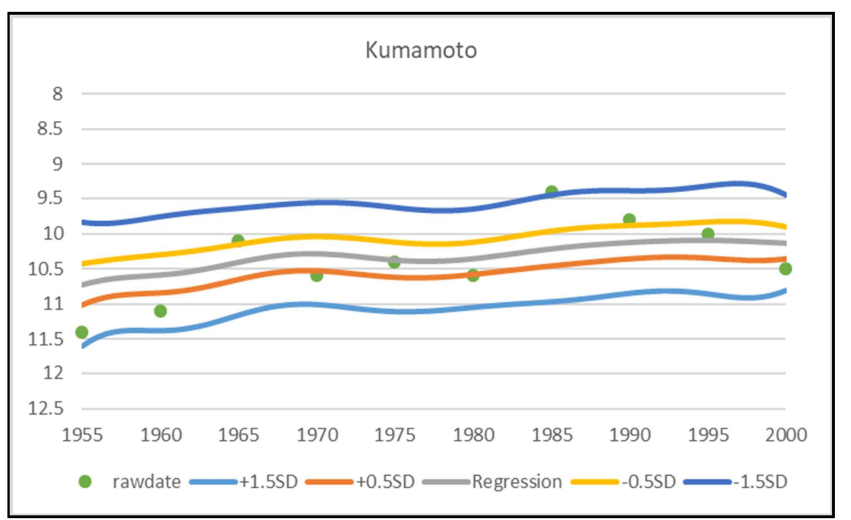

Figure 11. Investigation of the long-term trends in mean MPV age in girls (Kumamoto).

In Tokyo and other large cities, there was trend for earlier maturation from 1955 to 1970 , but afterward maturation trends were found to be average. In rural prefectures such as Aomori, maturation tended to be later from 1955 to 1970, but afterward a trend was seen for maturation to occur earlier.

\section{Discussion}

In this study, a 5-step evaluation chart was constructed from age at MPV of height for boys and girls from 1955 to 2015. In addition, by fitting the average age at MPV of height for each five years in each prefecture, we examined the relational composition with socioeconomic status by investigating whether there were differences in the change phenomena over time. The results showed a trend for earlier maturation in all prefectures for both boys and girls. The reason for this earlier maturation in boys and girls is thought to be that they received the benefits of economic growth from 1955 to today. Trends for taller height from the effects on height growth due to westernization of the diet with high postwar economic growth, and for younger age at MPV of height, were seen. It may be conjectured that this shows a trend for earlier maturation as a result. The reason for this is thought to be a relationship in which the higher GDP with economic growth led to expansion of the distribution network thanks to infrastructure development and other advances, from which consumption of foods with high nutritional value for protein and other nutrients increased with the importation of Western-style diets. 
This induced a DNA methylation phenomenon in the gene structure for height and produced a mini evolutionary phenomenon in which height grew across generations. As a result, growth in height was promoted and age at MPV of height became younger. In this way, a relationship is conjectured to exist between earlier maturation of age at MPV of height and economics.

In particular, we clarified the differences in the long-term change phenomenon from the multi-year span evaluation chart for each prefecture. Tokyo and other large cities tended to have earlier maturation from 1955 to 1970 , after which a trend for average maturation was confirmed. In rural prefectures such as Aomori, there was a trend for later maturity from 1955 to 1970 , but afterward there was a trend for younger ages. This demonstrates that many of the prefectures there were affected by high economic growth were those with large metropolitan areas, whereas prefectures that did not receive much benefit were those in rural areas. Matsumoto et al. derived results similar to the results of this study, and so more definitive results could be said to have been derived from the results of this study [14]. In addition, from these results, it may be that in prefectures thought to have been affected by high economic growth, the nutritional elements of dietary nutrition, and particularly the consumption of protein, affected physical growth. Yokoya derived similar results [15].

In contrast, it may be conjectured that there was a gradual effect with progress in the living environment and infrastructure development in prefectures considered to be in the aftermath of high economic growth. In addition, looking at the multi-year span evaluation chart since the late 1990s, it is conjectured that from around this time there was an effect from the ending of the period of high economic growth, based on the fact that there was no longer any distance in the rate of maturity between prefectures that showed earlier maturation from an early period and prefectures that showed a trend for earlier maturation in a somewhat later period.

\section{Conclusion}

In this study, using the wavelet interpolation method, we identified the age at MPV of height in each prefecture and examined the relational composition between high economic growth and the subsequent socioeconomic status in each prefecture. A trend for earlier maturation in both boys and girls was confirmed. This is thought to be the result of the effects of economic growth from 1955 to the present. The multi-year span evaluation chart in this study showed a trend for earlier maturation from 1955 to 1970 in Tokyo and other large cities, after which there was a transition to average maturation. In Aomori, Oita and other rural areas, there was a trend for late maturation from 1955 to 1970 , followed by a transition to earlier maturation. From these trends, there may be said to be a close relationship between physical growth and socioeconomic status. However, since the late 1990s the difference in maturation rate in urban and rural regions has disappeared. This may be from the effect of high economic growth coming to an end.

\section{References}

[1] Y. Matsuura, "Statistical growth and development study," Fumaidoshuppan, 2005.

[2] A. Kawabata, "Growth and development of Japanese people -Research on growth and development promotion phenomenon of youth-," Fumaidoshuppan, 1991.

[3] Y. Kani, K. Fujii, K. Kasuya, T. Watanabe, "High Economic Growth Period and Increase Phenomenon of Secular Trend in Infant Physical Growth," Production management: journal of Japan Society for Production Management Vol. 24 (1), pp. 81-86, 2017.

[4] F. Kikuta, M. Takaishi, "The Secular Trend of Standing Height in Adolescent Girls from Longitudinal Data," Bulletin of St. Luke's College of Nursing, Vol. 18, pp. 31-35, 1992.

[5] K. Fujii, "Verification Regarding Secular Trend of Height Growth and The Maximum Peak Velocity during Adolescence," International Journal of Sport and Health Science, Vol. 17, pp. 103-112, 2009.

[6] K. Fujii, Nho. Hosung, Kim. Seol-Hyang, "Confirmation regarding secular trend of physical growth in Korean school students by wavelet interpolation method: analysis from secular trend of age at MPV of height and weight," The Journal of Education and Health Science, Vol. 54 (2), pp. 129-140, 2008.

[7] S. Morichi, "The future of the land," Nikkei Inc., pp. 507-511, 2005.

[8] K. Fujii, "A scientific approach to growth and development-Physical information science for growth and development \& health." Sankeisha, 2006.

[9] Ministry of Education, Culture, Sports, Science and Technology "Annual Report of School Health Statistics Research,” 2015.

[10] K. Fujii, K. Kawanami, "An Analysis in regard to Relationship between Age at MPV of Height and Weight, and Its Sex Difference," Japanese Journal of School Health, Vol. 40, pp. 317-331, 1998.

[11] K. Fujii, Y. Matsuura, "Analysis of velocity curve for height by the wavelet interpolation method in children classify by maturity rate," American Journal of Human Biology, Vol. 11, pp. 13-30, 1999.

[12] K. Fujii, "Connection between Growth -Development and Mathematical Function-," International Journal of Sport and Science, Vol. 44, pp. 216-232, 2006.

[13] K. Fujii, T. Ishigaki, M. sho, Y. Saito, "Health science for life-span development -scientific search to health over life-span-," Kyorin-Shoin, 2008.

[14] K. Matsumoto, T. Mino, N. Nagai, H. Miyata, Y. Kudo, M. Shomoto, H. Takeuchi, S. Takeda, "Influence of Urbanization on Maximum Growth Age in Height for Each Prefecture in Japan," Japanese Journal of Hygiene, Vol. 35 (4), pp. 676-683, 1980.

[15] M. Yokoya, "Geographic Variation in the Body Size of Japanese Students and its Analysis by Mesh Climate Data," The Japanese Journal of Nutrition and Dietetics, Vol. 68 (4), pp. 263-269, 2010. 